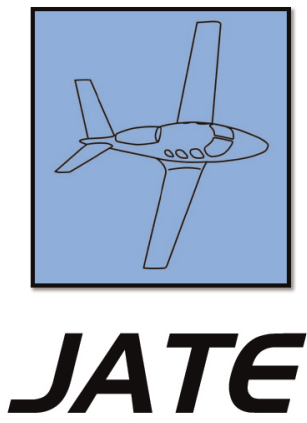

Journal of Aviation Technology and Engineering 2:2 (2013) 2-12

\title{
Modern Airline Pilots' Quandary: Standard Operating Procedures- to Comply or Not to Comply
}

\author{
Carrie N. Giles
}

Minnesota State University, Mankato

\begin{abstract}
Modern airline pilots are tasked every flight with the safe and efficient operation of highly automated airliners in today's complicated global and economic environments. Airlines have developed standard operating procedures (SOP) for normal, abnormal, and emergency operations. These procedures serve as a script for crews to follow. These procedures are designed by airlines to ensure that aircraft are operated in the (1) most safe, (2) most efficient, and (3) most on-time manner. For the most part pilots will comply with SOP, but when they (1) don't agree with SOP, (2) don't understand SOP or the risks associated with not complying with SOP, or (3) don't feel adequately trained to know what SOP is, it is difficult to motivate them to comply. Airlines have the means to measure compliance through Flight Operations Quality Assurance (FOQA) and Line Operations Safety Audit (LOSA). The purpose of this research is to determine if increased understanding, knowledge and awareness of the risk of noncompliance with SOP increase airline pilots' compliance with SOP. This research explores data from line checks at a major US airline that was gathered in pursuit of understanding what drives SOP compliance. Baseline data was gathered and analyzed to determine the top 12 noncompliant items. The airline provided training during the Human Factors module in each pilots recurrent training on Pilot Intentional Non Compliance (PINC). The training including developing pilots' understanding that while most Aviation Safety Action Program (ASAP) reports grant pilots immunity from legal action, if a violation is labeled PINC, ASAP protections do not apply. Further line checks were conducted after the pilots received the PINC training. The top 12 noncompliant items from the pre-PINC training group were compared to the same 12 items in the post-PINC training group. Significant improvement in SOP compliance was found in six of the 12 items tested. The results established that training pilots on the risk of PINC did significantly increase SOP compliance.
\end{abstract}

Keywords: airline, standard operating procedures, checklist, training, procedural compliance

\footnotetext{
About the Authors

Carrie N. Giles is a pilot for a major U.S. airline and a professor in the aviation department at Minnesota State University, Mankato. She holds a MS in Aviation and a BS in Aeronautical Studies from the Odegard School of Aerospace Sciences at the University of North Dakota. Correspondence concerning this article should be sent to carrie.giles@me.com.
} 
"Why? Because I said so." For the general population, these words provide very little motivation to comply with a request. However, in the black and white world of aviation, pilots are trained to follow the rules simply because they are the rules and the rules were made to be followed, and because the Federal Aviation Administration (FAA) and company policies and procedures "said so."

Checklists are completed step by step on every flight. Procedures for normal, abnormal and emergency operations are carefully developed and pilots are trained to follow them precisely. In fact, creative solutions are simply not allowed unless every other option has failed. In normal day-to-day line operations, Standard Operating Procedures (SOP) allow pilots who have never worked together before to safely operate complex flights. According to Dismukes, Berman and Loukopoulos (2007), "these written scripts establish the correct way to perform procedures and provide standardization across pilots. Standardization is crucial" (p. 2). Crews of two to four pilots are often brought together for the first time an hour before departure time and are expected to work together to provide the most safe, comfortable and profitable flight possible. Each pilot has a role and a script, and ideally, if SOP is followed, there should be very few surprises in normal, abnormal or emergency operations.

The knowledge of pilots' compulsion to comply with rules and regulations leaves airlines struggling to understand why data show that pilots are not dutifully complying with all SOP. Several recent accidents point directly to deviation from SOP as a primary cause.

In the fatal crash in Buffalo, New York of Continental Connection Flight 3701, a Bombardier Q400 that was operated by Colgan Air, the NSTB found that:

Contributing factors included his [pilot's] failure to monitor other warning signals of the plane's slowing speed, and violation of 'sterile cockpit' rules against casual conversation at low altitudes. The board also said Colgan had failed to spell out adequate procedures for crews to monitor air speed when icing is a risk (Trumbell, 2010, p. 90).

The wingtip damage of an American Airlines MD-82 on Flight 1402 was a nonfatal event in Charlotte, North Carolina:

A big question facing investigators is whether American's basic operational rules and procedures were followed. If complications crop up once an aircraft descends below 1,000 feet on an instrument approach, pilots generally are trained to break off the descent, gain altitude and circle back for another landing (Paztor, 2009, p. A6).

And, the investigation into the nonfatal crash of Continental Airlines Flight 1404, a 737-500 in Denver, Colorado, that departed the runway midfield during takeoff found that:
The captain's use of the nosewheel steering tiller was contrary to company procedures and neither of these late control inputs was an effective method for turning the airplane at high speed. The NTSB concludes that the captain's use of tiller and full right control wheel in the 3 seconds before the excursion likely resulted from acute stress stemming from a sudden, unexpected threat, perceived lack of control, and extreme time pressure (National Transportation Safety Board, 2010, p. 44).

Airlines are responsible to ensure SOP are safe and pilots understand and can comply with current SOP. The pilot's choice to deviate from or disregard SOP creates risk for the airline, passengers and crewmembers. According to Federal Aviation Regulations (FARs) and Airline Operation Specifications (Ops Specs), it is the pilot's obligation to comply (FAR/AIM 2012). This study explores the reasons for noncompliance and the effects of specific initiatives designed by airlines to improve pilots' compliance with SOP.

\section{Literature Review}

SOP are written, published and tested procedures that are expected to be universally and consistently applied within an organization. SOP should identify and describe the standard tasks and duties of a flight crew for each flight phase, including what to do and when to do it. Also, SOP should be simple, clear, concise and prescriptive (Aviation Glossary, 2010).

From the early days of ab initio training, modern airline pilots are trained in the use of checklists and other procedures defined as SOP. From the moment a crew is brought together in the flight planning room until postflight procedures are completed, a very specific series of steps are taken to dictate interactions between crew members. FAR 121.533 states "the Captain shall operate in accordance with FARs, Ops Specs [SIC] and airline procedures and policies except under circumstances provided for in FAR 121.557 [Captain's emergency authority]" (FAR/AIM 2012). Furthermore, operating manuals assign the Captain with the duty of ensuring the timely completion and accurate adherence to checklist procedures (Flight Operations Manual, 2011). When the Ops Specs provide SOP that are simple, clear, concise and prescriptive it is easy for a pilot to understand how to comply. However, sometimes SOP are not easy to understand and apply, and that makes compliance difficult (Dismukes et al., 2007). The overriding rules for airline operations are made by the Federal Aviation Administration (FAA), but SOP is carefully developed by each airline to take the airline beyond safety to improve efficiencies and reduce surprises in the cockpit. Sukenik (1998) found that, "Adhering to SOP leads to a maximum utility and, thus, to greater flight safety than deviating from it, even if an alternative procedure is equivalent as far as safety is concerned or seems to lead to greater utility" 
(p. 405). The airlines want their pilots to follow SOP because it has been proven to increase safety and save money.

Beyond the regulations, studies show that SOP should be adhered to in a pilot's pursuit of individual safety. Baker (2007) analyzed the effects of SOP compliance and found that, "Within an airline, management, operations, maintenance, training, equipment, and pilots must all be aligned. Accidents occur when breakdowns arise in the interactions of these components" (p. 186). Simply put, strict adherence to SOP helps each individual involved with the operation of a flight know what to expect. Baker's research showed clearly that, while SOP are not perfect, compliance with SOP make airlines safer, and noncompliance can lead to aircraft incidents and accidents. Furthermore, in analysis of 19 U.S. airline accidents in the period of 1990-2000, Dismukes, et al. (2007) found inadequate execution of highly practiced normal procedures under challenging conditions and deviation from explicit guidance or SOP as two of six common themes underlying the NTSB's label "pilot error" as an accident cause or contributing factor. They felt noncompliance due to lack of understanding or execution of SOP or blatant disregard for SOP are key problems that airlines must acknowledge and address in any attempts to increase SOP compliance.

\section{Advanced Qualification Programs}

In 1994 United Airlines Training Center developed the first Advanced Qualification Program (AQP). Today nearly all major U.S. airlines, along with growing number of U.S. regional airlines, participate in this voluntary program. The purpose of AQP is to encourage the use of innovative technologies, training and evaluation features to improve training performance. These programs are systematically developed, continuously maintained, and empirically validated as proficiency-based training systems. During a pilots career, one will experience initial (new-hire), transition (new airplane type), upgrade (new seat) and recurrent training. Recurrent training consists of simulator training to proficiency, crew resource management (CRM) and human factors (Hughes, 1995). Pilots are trained on the proper execution of SOP for all phases of flight in normal, abnormal and emergency operations. Evaluations are designed to ensure that understanding of SOP is demonstrated before the pilot is qualified for line operations.

Under AQP guidelines, captains are required to successfully pass a line check every 24 months. A line check is an observation of a pilot during line operations (Klinect, Helmreich, Murray, \& Merritt, 2003). During these line checks a check airman observes the Captain and assesses SOP compliance. These line checks are jeopardy events, meaning the pilot must pass or be removed from line flying for training to resolve deficient areas. Some of the Microscope Line Checks (MLC) referred to later in this study were done during line checks required by AQP.

\section{Line Operations Safety Audit}

Besides the AQP required line checks, airlines employ a safety tool known as Line Operations Safety Audit (LOSA). These cockpit observations are similar to line checks as they are gathered during normal flight operations, but their purpose is different:

In the most general of terms, LOSA is similar to getting your cholesterol checked during a routine examination...it provides a diagnostic snapshot of safety performance. It uses cockpit observations collected in normal operations to provide a profile of safety strengths and weaknesses. Similarly, the onus is on the airline to respond to the data and make change if necessary." (Klinect et al., 2003, p. 2).

\section{Learning Theory}

Since the purpose of this research is to evaluate experienced pilots' compliance with SOP, it is important to understand how pilots learn following initial training. While each new learning experience presents challenges of different degrees, professional pilots are expected to perform well in all aspects of training. In line operations, pilots are expected to adopt and comply with company SOP. This requires pilots to overcome any bias, including any personal dislike or disagreement with SOP (Dismukes et al., 2007).

Understanding how pilots learn and make decisions is key to developing training on SOP. SOP is not necessarily developed to bring pilots to the best choice, based on the pilot's experience, but to a prescribed choice. All procedural changes require the creation or alteration of SOP and the training for pilots to understand what is now expected of them. The airlines are very experienced and very good at making SOP for all areas of operation, and as new procedures are implemented, a checklist normally accompanies them (Sukenik, 1998). The concern arises when the FAA sets the minimums for training requirements, and the standards are influenced by cost cutting initiatives. Dismukes et al. (2007) found "inadequate knowledge or experience provided by training and/or guidance" (p. 298) was a factor in more than a third of the 19 accidents studied. A critical component of these accidents involved pilots who found themselves in "challenging situations for which they had received training, but the experience they received from that training was of inadequate fidelity to the actual situation, inadequately detailed, or incomplete" (p. 298). In these accidents the authors discovered the pilots completed the training, but the airlines' efforts to ensure that pilots understood SOP to the point they would be comfortable executing new procedures simply fell short. The authors go on to explain that if airlines were more aware of the limitations of their 
training, they could make modifications and train pilots to make well thought out decisions that utilize SOP based on their solid understanding of how to apply the new SOP to real-life situations.

\section{Pinc and Punc}

James Huntzinger, the former Vice President of Safety, Security \& Compliance at Korean Air has been credited with coining the terms procedural intentional noncompliance (PINC) and procedural unintentional noncompliance (PUNC) (Agur, 2007). Quite simply, these acronyms are used to label behavior as pilots' unintentional or intentional deviation from company prescribed SOP. The Air Safety Foundation (2007) reported that a review of accidents involving professionally flown aircraft shows that four out of five events included PINC or PUNC by pilots. Additionally, "PINCs and PUNCs are reduced dramatically when an effective safety culture exists." (Agur, 2007, p. 13). One might conclude the way to reduce PINCs and PUNCs is to increase the effective safety culture at an airline. Simple as that sounds, U.S. major airlines are already incredibly safe and have effective safety cultures in place (Snyder, 2007).

It has already been established that airline safety records are commendable and current regulations motivate operators to avoid situations where they could be violated by the FAA, yet PINC events are regular occurences. Huntzinger (2006) summarized the following:

"PINC events typically involve crews who have been flying most of their adult lives — veteran aviators who train on simulators once or twice a year; attend. recurrent training classes that cover procedures, FARs, limitations and other best practices; practice CRM; ride with check airmen; and so on. In short, they clearly know the rules and regulations, yet...intentionally violate them" (p. 42).

Huntzinger describes the three elements of a PINC event as: (1) a reward for the violator; (2) knowledge of the associated risk; (3) consideration of how one's peers will react. If a pilot is able to determine that, for example, the reward of an on-time arrival (getting home sooner) is worth the risk of an unstabilized approach (that by SOP should result in a goaround) and in their opinion they determine that continuing the approach and landing would be considered safe enough by their peers, even if it violates SOP, and even though they know better, their safe enough landing has all the elements required for PINC. "They [PINC] are often the result of wellmeaning pilots trying to do their job but willfully taking risks to achieve what should be a secondary goal, 'completing the mission.'” (Agur, 2007, p. 36)

PUNC can be considered a SOP violation that is less deliberate in nature, but it is the result of a lack of information or understanding that results in a pilot unknowingly violating SOP. The pilot's responsibility is to comply with company SOP, and the airline is responsible to train the pilots who operate their aircraft. An effective airline safety culture strives to inform pilots of ever evolving SOP, but this is not an easy task. Anthony (2009) summarizes: "Aviation is inherently a dynamic and everchanging industry that is constantly producing hazards even as it strives to reduce them" (p. 42). The task of finishing the work of SOP development so that pilots can be perfectly informed of SOP is never complete, and PUNC is often the result of a lack of training by the company and thereby pilot's efficiency in understanding of SOP.

\section{Research Questions}

The purpose of this research is to determine if increased understanding, knowledge and awareness of the risk of noncompliance with SOP will increase airline pilots' compliance with SOP. As their experience grows, their adversity to high-risk may cause them to avoid troublesome situations. Often times their intelligence drives them to make the wisest, most prudent choice as they strive for safety. A natural extension to understanding pilot personalities might be to assume that they enjoy the establishment of SOP and are excited to follow it to the letter. Quite the contrary, pilots are not content with the way labor has been treated in "the airline industry's...most volatile period in the past 20 years" (Goodman, 2008, p. 14). This frustration may serve as a distraction resulting in attention being diverted from SOP (Dismukes et al., 2007).

Since September 11, 2001, uncertainty has ruled airline aviation, and when it comes to pushing for compliance, pilots have a tendency to push back. A quandary is defined as a state of doubt or uncertainty, especially with regard to the choice of alternatives (Quinion, 2008). In the case of PINC, the pilots' know SOP but choose to ignore it, where in the case of PUNC the SOP may not be clear or properly understood. In every case pilots are tasked to comply, even if they are unsure what a specific SOP compliance requires or don't like the SOP. Perhaps this is why pilots find themselves in a quandary regarding SOP compliance - they are conflicted. SOPs are developed by airline management and experienced line pilots in the safety and comfort of an office. Safety, trainability and what is best for the company are all factors in how SOPs are determined. Along with experience and past practice, they consult the manufacturer's airplane flight crew operating manual and other sources to determine what SOP is best. New initiatives result in new procedures and it is sometimes difficult for pilots to keep track of which procedure is the current procedure, especially when the reason for the change to SOP is not explained or understood. Pilots may feel that the SOP prescribed for a given scenario is unsafe, complicated, or simply unnecessary. Pilots are opposed to change, especially when the procedure being replaced was, in their 
opinion, was at least as safe, if not more safe than the new SOP (Dismukes et al., 2007).

Also, because of Captain's authority, pilots mistakenly believe their certificate entitles them to disregard SOP if they have determined that their way is "more safe." This is true in the application of 'recommended practices', the term applied to specific techniques trained by the airline. When it comes to recommendations, pilots can adopt the recommended technique or develop their own (Aircraft Operating Manual, 2010). For SOP, except in an emergency situation where an emergency has been declared, failure to comply with SOP is a violation of the certificate they hold, which requires them to operate according to company policies and procedures (FAR/AIM 2012). Therefore, pilots may believe that the company prescribed way to operate a jet and the absolute safest way to fly are not equal. In a specific airline's AQP, recurrent training time is often limited because the goal is to meet the minimum requirements and keep costs low. According to Rigner and Dekker (2000), today's airlines are sometimes faced with limited training time available, and their chosen training approach may not allow instructors to elaborate where need is shown because of prescribed tasks that must be accomplished. In other words, there is a lot to cover in a limited amount of time, which often results in training deficiencies that may or may not show up in Line Oriented Flight Training (LOFT) or line checks. Updates to current procedures are distributed to pilots by bulletins, and amended SOP are sometimes trained by textual description alone. Pilots are expected to maintain a firm understanding on the execution of current SOP, often times without receiving any training on it. The amount of information and training provided with changes to SOP may not be adequate.

Several authors state that the lack of understanding of the implications of disregarding SOP is a factor in a pilot's decision to violate SOP. Before FOQA programs, airlines were largely unaware of the specific configurations their aircraft were operated by a particular pilot on a given day. With FOQA, the gatekeeper can link the pilot to flight performance data through all phases of flight. Another factor is the protection of the ASAP program, which protects pilots as long as the violation was unintentional and not in violation of SOP. Pilots may not have adequate understanding of the consequences of PINC, which include being violated by the FAA because the immunity of the ASAP programs will not extend to intentionally risky behavior. The truth is "they" (the company and FAA) know what pilots are doing on every flight and the responsibility to comply with SOP is not only required during line checks. Therefore, pilots may not understand the risk associated with a decision to disregard SOP. This is a new change, and once pilots are trained on the risk associated with noncompliance with SOP, compliance may increase.

The research questions posed by this study are:

1. Do microscope line check findings differ following instruction on PINC and PUNC as compared to preinstruction findings?
2. Do the top 12 "non-standard" items on the microscope line check improve in rank amongst top 12 items following instruction on PINC and PUNC?

\section{Methodology}

This research is to determine if increased training of the risk of noncompliance with SOP will increase airline pilots' compliance with SOP. To establish a need for further study, LOSA checks were accomplished at a major U.S. airline during January of 2010. As a result of the LOSA checks, it was determined that more emphasis and education were needed concerning SOP compliance. Microscope line checks (MLC) were conducted on 308 flights in January and February of 2010. Check airmen were assigned to observe on randomly selected routes to collect the data and complete the checks. These MLC were classified as line checks, not LOSA, and therefore if a pilot were to fail the MLC they would be disqualified and sent for training in accordance with AQP. After the high frequency MLC in January through February of 2010, the airline elected to continue using the same survey on all scheduled line checks required by AQP. Additional data was collected through March 2011 and is also used in this research. All data were recorded anonymously and tested against specific changes to airline training and policies to determine effect on pilot's SOP compliance. Finally, the Institutional Review Board at the University of North Dakota reviewed and approved the project including the survey questions, proposed sample, and research methods.

The checks utilized a standardized survey of 60 different SOP items. The survey items were broken down by phase of flight. Each phase of flight included any checklists to be completed, actions to be taken and any other specific SOP actions required. There was also a section for general SOP items, for example "Appearance" or "Point and Shoot used for all altitude changes". Each item was scored either as "Standard," "Nonstandard," "Not Applicable" or "Not Observed." The check airmen performing the MLC were asked to record comments on each of the "Nonstandard" items to establish the reason for the discrepancy. To understand the specifics of each occurrence of noncompliance, the researcher recorded and analyzed the comments from each of the flights conducted in January and February of 2010. While this qualitative data was not used to determine any trends of noncompliance, it was used to determine which specific initiatives and procedural changes should be tested for potential effect on SOP compliance.

Specific training on the consequences PINC was added to the Human Factors training module that was presented to pilots during initial, transition, upgrade and recurrent training. By August 31, 2010, all participants had completed this training.

Participants were current and qualified flight crews at a major U.S. airline. MLC checks were randomly conducted 
on flights in both domestic and international flight operations during January, February and March. Additional MLC data was gathered during the next 12 months during AQP required line checks. With few exceptions, all flight crew members hold a type rating on the aircraft flown. The check airmen who conducted the MLC are also type rated, current and qualified captains on the equipment checked. Participants remained anonymous except for the generalized demographic data queried at the beginning of the survey.

The study author notified and received written permission from management of the respective airline to utilize data collected from the survey. The Institutional Review Board at the University of North Dakota reviewed and approved the project survey questions, proposed sample, and research methods.

The one area of concern for bias is the potential for falsepositives on items found to be "Standard" SOP performance. The MLC is a testing event, and therefore it is impossible to know if the pilots exhibited "standard" behavior because they always follow SOP, or if they are complying with SOP because they are being watched and face de-qualification if they fail to comply with SOP. It is unrealistic to expect that pilots will demonstrate the same behaviors they would if the check airman were truly an unknown presence. Therefore, any analysis will be limited to application to SOP compliance with a check airman in the cockpit, as it is impossible to know if the same compliance will carry over to line operations (Helmreich, 2003).

Additionally, some of the participants who were checked during the random MLC used to establish the baseline for SOP compliance may have attended Human Factors training prior to their MLC. Statistically, only $14 \%$ of line pilots experienced this training during this period. Because the MLC were conducted as the company initiative was launched, no data without this bias existed. The Human Factors training on PINC would have biased them towards SOP compliance, so using those numbers as a baseline is more conservative than to have purely unbiased numbers.

The data were analyzed to determine if the effect on SOP compliance is significant. The baseline data were analyzed to determine the top 12 areas of noncompliance. Those data were ranked and compared with the data collected during the months after the PINC training was presented to all pilots to determine what effect it had, if any, on SOP compliance rates.

The data set was divided into two groups, pre-PINC training for the data collected in January and February of 2010, and post-PINC training for the data collected after September 1, 2010. The data from the pre-PINC training flights were ranked based on the number of 'non-standard' events in each of the 60 areas tested. The top 12 areas of noncompliance from the pre-PINC training group were analyzed in this study.

The first group of data, labeled pre-PINC training, was from the period before all subjects experienced the Human
Factors module containing PINC training. These data were gathered from 306 randomly selected flights. Of the 60 areas tested, the top 12 areas of non-compliance from January and February 2010 MLC (pre-PINC training) were ranked in Table 1.

The second group of data, labeled post-PINC training, was collected during flights that were flown after each line pilot at the airline had received PINC training during the last nine months. These data were gathered from 289 flights during which AQP required line checks were being accomplished. Of the 60 areas tested in the survey, the top 12 areas of noncompliance from the September 2010 through March 2011 MLC (post-PINC training) were analyzed.

\section{Statistical Analysis}

The data groups were compared using chi-square analysis to determine any significant change in compliance levels between pre-PINC and post-PINC training time periods.

\section{Data Summary}

Of the top 12 noncompliant areas analyzed for relationship, six of the 12 showed increased compliance with the specific area of SOP. The greatest difference between the pre-PINC training and post-PINC training groups was in non-standard appearance.

\section{Results and Discussion}

The data show that in six of 12 specific areas studied, pilots who have been exposed to training on PINC will perform SOP in a manner that the company will grade "standard" on line checks. In nine areas $(n=12)$ there was

Table 1

Top 12 non-standard items ranking

\begin{tabular}{|c|c|c|}
\hline & $\begin{array}{c}\text { Pre-PINC } \\
\text { Training Rank }\end{array}$ & $\begin{array}{c}\text { Post-PINC } \\
\text { Training Rank }\end{array}$ \\
\hline Non-standard Item & $(\mathrm{n}=306)$ & $(\mathrm{n}=289)$ \\
\hline Climb speed policy compliance (29) & 1 & 1 \\
\hline $\begin{array}{l}\text { Standardized descent speed } \\
\text { compliance }(44)\end{array}$ & 2 & 6 \\
\hline Appearance (63) & 3 & 12 \\
\hline $\begin{array}{l}\text { "Point and Shoot" for all altitude } \\
\text { changes }(65)\end{array}$ & 4 & 7 \\
\hline Before Takeoff checklist (26) & 5 & 3 \\
\hline Before Taxi/Taxi checklist (18) & 6 & 8 \\
\hline Takeoff briefing accomplished (13) & 7 & 10 \\
\hline $\begin{array}{l}\text { (G)FMS route/legs verification } \\
\text { check (12) }\end{array}$ & 8 (tie) & 4 \\
\hline After-Landing - Taxi checklist (57) & 8 (tie) & 11 \\
\hline Parking checklist (61) & 10 & 9 \\
\hline Before Starting Engines checklist (16) & 11 & 2 \\
\hline Cruise Checklist (31) & 12 & 5 \\
\hline
\end{tabular}


Table 2

Non-standard results

\begin{tabular}{|c|c|c|c|}
\hline Non-standard Item & $\begin{array}{c}\text { Pre-PINC } \\
\text { Training } \\
\text { Observed/expected }\end{array}$ & $\begin{array}{c}\text { Post-PINC } \\
\text { Training } \\
\text { Observed/expected }\end{array}$ & Sig. \\
\hline Climb speed policy (29) & $48 / 38.8$ & $27 / 36.2$ & 0.023 \\
\hline $\begin{array}{l}\text { Standardized descent speed } \\
\text { (44) }\end{array}$ & $28 / 20.4$ & $12 / 19.6$ & 0.012 \\
\hline Appearance (63) & $22 / 11.2$ & $0 / 10.8$ & 0.000 \\
\hline "Point and Shoot" (65) & $21 / 15.7$ & $10 / 15.3$ & 0.051 \\
\hline $\begin{array}{l}\text { Before Takeoff checklist } \\
\text { (26) }\end{array}$ & 20/19.0 & $17 / 18.0$ & 0.741 \\
\hline $\begin{array}{l}\text { Before Taxi/Taxi checklist } \\
\text { (18) }\end{array}$ & $18 / 12.9$ & $7 / 12.1$ & 0.036 \\
\hline Takeoff briefing (13) & $16 / 10.8$ & $5 / 10.2$ & 0.022 \\
\hline $\begin{array}{l}\text { (G)FMS route/legs check } \\
\text { (12) }\end{array}$ & $15 / 15.3$ & $15 / 14.7$ & 0.901 \\
\hline $\begin{array}{l}\text { After-Landing - Taxi } \\
\text { checklist (57) }\end{array}$ & $15 / 9.3$ & $3 / 8.7$ & 0.006 \\
\hline Parking checklist (61) & $14 / 10.3$ & $6 / 9.7$ & 0.088 \\
\hline $\begin{array}{l}\text { Before Starting Engines } \\
\text { checklist (16) }\end{array}$ & $13 / 15.9$ & 18/15.1 & 0.277 \\
\hline Cruise Checklist (31) & $12 / 12.9$ & $13 / 12.1$ & 0.717 \\
\hline
\end{tabular}

an overall improvement in SOP compliance. In two areas where the SOP compliance did not improve, the item tested was a checklist with multiple items, and therefore multiple places where the non-standard behavior could have been displayed. Further research into the qualitative section of the MLC form would be useful to determine the cause of the "non-standard" grading.

Checklist items accounted for two of the six areas with significant improvement. In both the Before Taxi/Taxi Checklist $\left(\chi^{2}(1, \mathrm{~N}=591)=4.418, \mathrm{p}<0.5\right)$ and the After Landing-Taxi Checklist $\left(\chi^{2}(1, \mathrm{~N}=586)=7.439, \mathrm{p}<\right.$ $0.5)$, pilots who experienced training on PINC were found to perform better than pilots who had not received PINC training. This is noteworthy because in the Before Taxi/ Taxi Checklist and After Landing - Taxi Checklist there are 11 (approximately - depending on fleet type) items on the checklist, and therefore 11 places to find oneself graded "non-standard" by a check airmen. The specific checklist items with noted improvement each consist of over 10 individual checks which could each result in noncompliance. Additionally, the Before Taxi/Taxi checklist was introduced in 2009 in order to achieve fleet standardization. Before that time, fleets only had Taxi checklists. The improvement in compliance could also be attributed to pilots becoming familiar with new procedures during the recurrent training by the check airmen emphasizing the new change, something that occurred during the same training cycle they received their PINC human factors training.

Non-standard descent speed compliance was number two in the pre-PINC training "non-standard" rankings, and showed improvement to number six in the post-PINC training rankings $\left(\chi^{2}(1, \mathrm{~N}=577)=6.239, \mathrm{p}<0.5\right)$. The reason for this is twofold: first, "minimum descent speed checked" is an item on the first section of the Cruise
Checklist. It is a simple entry (cruise mach/aircraft specific descent speed) called for at a quiet part of the flight (level off), and the checklist cannot be called "complete" until it is entered. Second, PINC training specifically addressed checklist completion. It is interesting to note that the number 12 "non-standard" item in the pre-PINC training rankings was the Cruise Checklist, which showed no improvement in SOP compliance in post-PINC training group, and it actually had one more occurrence of noncompliance in a smaller number of total flights in the post-PINC training group.

In terms of ranking, the Cruise Checklist $\left(\chi^{2}(1, \mathrm{~N}=\right.$ $584)=0.131, p>0.5$ ) had the second worst 'decline' in ranking between the pre-PINC training ranking and the post- PINC training ranking, rising from 12th worst area of non-compliance to 5th. "Minimum Descent Speed Checked" is on the Cruise Checklist. Further research into the comments on "non-standard" grades on non-standard Cruise Checklist, is required to understand the reason for the "nonstandard" marks.

Non-standard climb speed policy compliance showed significant improvement from the pre-PINC training group to the post-PINC training group, yet it still remained the number one area of noncompliance in the post-PINC training group $\left(\chi^{2}(1, \mathrm{~N}=586)=5.205, \mathrm{p}<0.5\right)$. The airline introduced the climb speed policy in 2009 in the interest of minimizing fuel burn and standardizing fleets. Unlike non-standard descent speed, "climb speed" is not an item on any checklist. It is supposed to be entered into the flight management computer during the preflight, and it is covered under the umbrella of the "CDU - Checked" response on the Before Starting Engines Checklist. Before the policy was introduced, pilots used a variety of recommended practices to program their climb speeds (i.e., flight plan climb speed, transitioning to cruise mach number), but there was no existing SOP for the entry of a climb speed. The new climb speed policy was distributed by pink bulletin as an amendment to the Aircraft Operating Manual, and at first a reminder was printed out in the appended messages portion of every flight plan. This "reminder" was removed at some point after the pre-PINC training group MLCs took place. The Standard Climb Speeds are currently located in the aircraft operating manual in the Climb - Cruise - Descent section, something not normally accessed during normal preflight procedures.

Non-standard takeoff briefing accomplished was unique as it was the only item in the top-12 non-standard ranking with an expanded definition of which SOP items check airmen should be looking for. The description lists "Taxi Route, Hot Spots, SID, FM II special procedures, engine out, terrain considerations (MEA, MSA, Grid MORA), Transition Altitude, takeoff." The results showed that pilots who had received PINC training were more compliant with SOP than the pre-PINC training pilots $\left(\chi^{2}(1, N=587)=\right.$ 5.266, $\mathrm{p}<0.5)$. This validates the researchers belief that PINC training will increase SOP compliance, but in the 
pursuit of understanding the SOP for a takeoff briefing the researcher found no place in the Aircraft Operating Manuals or in the Flight Operations Manual where all these requirements are listed as SOP. In fact, all that is included about takeoff briefings in the Aircraft Operating Manual is a requirement to designate the pilot-flying, brief rejected takeoff considerations and any other contingencies (if applicable) (Aircraft Operating Manual, 2010).

Of those listed on the MLC form, standard instrument departure (SID), engine out, terrain and takeoff are the only ones that the researcher could find in the governing books of the airlines SOP. It would be expected that a pilot could decide that in order to effectively brief terrain considerations, the minimum safe altitude (MSA) should be briefed, or that it is wise to brief the planned taxi route, but that would be considered recommended technique, not SOP. The term "Hot Spots" is not found in any of the manuals, nor is there any requirement to brief the taxi route. International Civil Aviation Organization (ICAO) defines a hot spot as a location on an airport movement area that has historically been at high risk for collisions or runway incursions (Federal Aviation Administration, 2011). These areas are highlighted on airport charts to increase awareness for pilots and drivers. The qualitative comments from the pre-PINC training group explain why the "non-standard" mark was given included, "Captain did not brief 'hot spots' along planned taxi routing" and "No taxi route brief." It seems there might be some confusion in the training department as to what the official SOP is for the takeoff briefing. This exemplifies the need for simple, clear, concise and prescriptive SOP so that everyone, pilots and airline training departments, understands what is expected (Aviation Glossary, 2010).

The largest improvement in SOP compliance was found in nonstandard appearance. $\left(\chi^{2}(1, \mathrm{~N}=579)=22.169\right.$, $\mathrm{p}<0.5)$. This standardized residual far exceeded $1.96(\mathrm{z}=$ 3.2 ) and the actual $p<0.01$. While it is possible to draw the conclusion that PINC training had an incredible effect on pilots appearance, it is interesting to also note that the flight department at this particular airline distributed a letter to all pilots signed by a majority of the chief pilots indicating that the culture of uniform compliance is changing. Another possible contributing factor was a change in leadership that was accompanied by a lessrestrictive hat and tie policy "rumor". That rumor became official in a recent revision to the carrier's flight operations manual. Analysis of the pre-PINC training data comments to understand how many were hat and tie related might reveal fewer non-standard appearance scores if the current policy were applied to pre-PINC training data. Regardless, the airline can be satisfied that they met the seemingly unachievable goal of perfect compliance with SOP in this one area in the post-PINC training line checks.

Of the remaining items to be discussed, three of them are checklists that showed no significant difference between the pre-PINC training and post-PINC training data. In fact, the non-standard marks on the Before Starting Engines, Before Takeoff and Parking Checklists all had worse rankings on the post-PINC training top-12 than on the prePINC training top-12. $\left(\chi^{2}(1, \mathrm{~N}=591)=1.183, \mathrm{p}>0.5\right)$.

The Before Starting Engines Checklist compliance item was ranked 11th worst pre-PINC training to 2 nd worst postPINC training, the greatest increase in rank of all items. The researcher believes that due to the large number of items on a checklist, it is easy to find something non-standard which would deem the entire checklist non-standard. On one fleet, the Parking Checklist's non-standard comments involved six different checklist items, three of which were only marked non-standard on a single flight. $\left(\chi^{2}(1, \mathrm{~N}=587)=2.905\right.$, $\mathrm{p}>.05$ ).

It is important to study further the source of these nonstandard marks and determine which areas can be focused on to improve pilot's understanding of SOP on normal checklists. The final item for discussion is item 12, nonstandard (G)FMS route/legs verification check. Regarding item 12 , the SOP for this is not new $\left(\chi^{2}(1, \mathrm{~N}=589)=\right.$ $0.015, \mathrm{p}>0.5)$. This item's ranking moved from 8th least compliant area to 4th least compliant, the third worst decline in rank. The researcher found the SOP for this item to be fairly consistent in all fleets, with variations in the types of (G)FMS the only reason for between-fleet differences. The interesting finding about this data was that of the 30 flights found non-standard, 25 of them $(83.33 \%)$ were flown on domestic routes. A possible explanation for this finding is that international routes are complex and often flown over areas without reliable ground based navigational aids or radar coverage, the flight plan is checked against the (G)FMS for accuracy many times. Domestic flights are almost always in radar coverage, routes are familiar, and while the chance for violation is just as prevalent as it is on international routes, the fear of a gross navigational error fine simply doesn't exist. This theory could be researched further to understand if there is a significant difference in (G)FMS route/legs check compliance between domestic and international flights and, if so, explore the comments to further understand potential solutions to increase domestic pilots awareness of how to comply and the risk of noncompliance.

\section{Recommendations}

Due to the significant findings of improvement in the post-PINC training group in six of the 12 areas tested, this researcher feels that PINC training should continue to be a part of human factors training. Additionally, the airline should continue to look for new ways to increase pilot's understanding of the consequences of choosing to disregard SOP. As of September 1, 2010, Captain's decision making training was added to the PINC training. However, in order to increase the safety culture the airline should provide 
assertiveness training to the First Officers. This training should involve both Captains and First Officers with the goal of establishing roles and expectations. First Officers must respect the role of the Captain while helping the Captain comply with SOP, and alerting the Captain to any deviation from SOP is part of that. This training has the potential to bring the CRM aspect of SOP full circle and this step is vitally necessary in pursuit of a more SOP compliant operation and improvement in the overall safety culture of the airline.

To simplify SOP compliance, a Quick Reference Card specific to each fleet should be developed. In the case of Climb Speed compliance, the card would serve to make complicated Climb Speed tables easily available for pilots to crosscheck every flight. This card could be used as a tool to help pilots comply with all items required on the Takeoff briefing. This tool should also include similar lists for Crew Change briefings for 3- or 4-pilot crews and for the Approach briefing called for on the Descent Checklist. Aircraft limitations as well as other recommendations from line pilots could be added as well. Research into the causes of non-standard procedure compliance in the MLC comments section could reveal additional items to include on the Quick Reference Card. The card should be administered not as a recommended tool, but as SOP for all crewmembers to reference the card, at least in the case of multiple item briefings.

Because six of the top- 12 areas of noncompliance were checklist related, a renewed focus on checklist discipline would be recommended to improve checklist SOP. There were a number of "no 'checklist complete' call out" and "incorrect response to a couple of items" qualitative remarks on all the checklists that made the top-12. Checklist discipline is something that some pilots make a daily practice of, while others make sure they brush up on it for whenever they need to go to training. If the pilots are encouraged in training to make line operations checklist discipline just as stringent as it is in training, the culture could shift quickly to be more SOP compliant. First Officers should feel compelled to correct their Captains if they say, "Set" when they should say, "Checked," for example, and Captains should correct First Officers on incorrect responses to challenge and response items when they are the pilot monitoring (PM). The responsibility for good checklist discipline falls on both Captains and First Officers, and the one holding the card and reading the checklist has all the answers in hand.

One of the biggest challenges for many U.S. major airlines is that they essentially operate two different airlines, one domestic and one international. They have short-haul and long-haul flights and a variety of fleets enlisted to accomplish the goal of safely moving people. In the goal of standardization, these differences cannot be ignored. Nonstandard (G)FMS route/legs verification check, brought to light a difference between compliance rates domestic and international flights. If it is the airlines plan to standardize fleets in this operating area, it is important that this procedure is highlighted and the reason behind checking the paper flight plan with the electronic flight plan is brought to light. Pilots are frustrated when they are asked to do something because it is SOP, especially when it seems to be excessively redundant for routes they routinely fly. There are many reasons why this route/legs check is important, and the application of this procedure to domestic flights makes sense because there is a potential for mistakes if the procedure is not conducted properly. The skies are getting more populated, and ATC separation requirements are shrinking. Educating pilots on the "how?" and "why?" of a procedure that they may have deemed unnecessary could shift their thinking.

The final recommendation of the researcher is in all development of training and new SOPs, differences between fleets and pilot's aversion to change must be acknowledged and addressed to effectively increase SOP compliance.

\section{Conclusion}

The question as to whether increased understanding, knowledge and awareness of individual airline SOP and the risk of noncompliance with SOP increase airline pilot's compliance with SOP has been only partially explored by this research project, and further research is required to more fully answer the question. Because the data was collected on multiple fleets in both domestic and international operations on long-haul and short-haul flights, further investigation comparing data in these groups could lead to better understanding of specific areas where SOP compliance rates may differ. This research could serve to better explain the conditions under which greater rates of noncompliance exist in certain operations, thereby allowing training to be designed to directly address the problem area. This would help to avoid blanket solutions that, applied company wide, are often rejected by the groups not exhibiting the noncompliant behavior. Additionally, the airline should consider a study of their current checklist procedures to determine if there is any room for improvement or change.

It is also recommended that the airline use future data to understand the effect of non-training events on SOP compliance. The PINC training that pilots received that was tested for this research project was an example of an external motivator for compliance. If pilots don't comply with SOP, they risk being violated. This external motivator, while shown by this study to be effective, is defined as a negative motivator. The U.S. Navy publishes this about the use of negative motivation on subordinates: "Fear activates such negative incentives as threat of punishment or restriction of personal needs. Negative motivation, however, often destroys morale; and effectiveness will decline 
as morale declines. Long-term or frequent use of negative motivation is self-defeating" (U.S. Navy, 2010, p 4-17). Future research into the effects of positive external motivators like improved working conditions due to settled union labor disputes, positive corporate financial reports, and the announcement of growth with new airplanes, route, or hiring of new pilots should be conducted. This research could reveal if improved morale and the possible effect of a less distracted cockpit environment could lead to an increase in SOP compliance. Data gathered during times of expansion could compare new Captains to Captains who have been in the left seat for five years or more to determine who is more SOP compliant. These and many other questions could be analyzed to better understand what motivates pilots to comply with SOP.

Due human error, it is not possible for airlines to ever achieve $100 \%$ compliance with SOP, no matter how much training, clarification and understanding pilots have of existing SOP. Pilots will make mistakes, but the determination to pursue perfect SOP compliance should be a part of a pilot's commitment to professionalism just as primum nil nocere, "first, do no harm," is a fundamental part of physician's ethics training in medical school. It is an attitude of excellence, a foundation that serves to underlie the best, most safe operation every time pilots are charged with the command of an aircraft full of trusting passengers.

The amount of man-machine interaction required in the operation of highly automated aircraft flown by airlines today is negligible when compared to the virtually nonautomated 707 or even the 727 . This has reduced the need for pilots to 'do' and increased the need for pilots to 'monitor'. Bhana (2010) states, "The paradigm shift is significant, as it requires a different pilot skill set to be added to the traditional 'stick and rudder' skills" (p. 14). Couple this threat of complacency with FOQA and the threat of PINC and PUNC, and it is vividly apparent that today's airline pilots are operating in a different world in 2012 than they were even 5 years ago, and it is drastically different from the environment 15 to 35 years ago when most of today's U.S. major airline pilots learned to fly. Acknowledgement of that change requires understanding of the implications that accompany FOQA, PINC, PUNC and automation, bringing to light the fundamental need for SOP compliance.

Aristotle once said, "Excellence is an art won by training and habituation. We do not act rightly because we have virtue or excellence, but we rather have those because we have acted rightly. We are what we repeatedly do. Excellence, then, is not an act but a habit" (n.d.). John Hale, the Vice President of Flight at American Airlines has been credited with coining the phrase 'aggressively safe.' In his introduction to the Flight Operations Manual he states, "We just do not have the luxury of being anything but excellent every single time we climb into the cockpit"
(Hale, 2011). Aggressively safe is an attitude he asks his pilots to adopt every time they fly, a proactive approach to flying in pursuit of preparation for the unknown threats that absolutely exist. He believes that, like in defensive driving, we must pursue safety throughout all phases of flight because the threat of complacency is real. SOP compliance is the foundation to aggressively safe operations.

If airlines can provide pilots with SOP that are simple, clear, concise and prescriptive and provide pilots with the training to understand the reason for SOP and how to successfully apply it and tools to make compliance easy, they will have done their part. This research established that training on the "why" behind the "because I said so" that is the risk of PINC did increase SOP compliance. While SOP compliance is the goal, if pilots receive training, clarification and achieve understanding of the 'why?' and 'how?' of SOP, they will be far more motivated to do the right thing. "Because I said so," works, but "because it is the right thing to do," changes the motivation from fear to one where the satisfaction of complying with SOP because they understand and can justify it as the right thing to do positively motivates pilots and engages them as professionals to be an important contributor to the airline's safety culture.

\section{References}

Agur, P. (2007). Discipline as antidote. AeroSafety World, 2(2).

Agur, P. (2007). High culture: In corporate aviation. AeroSafety World, 2(6), 13-21.

Anthony, T. (2009). Safety culture: SMS on wheels. AeroSafety World, $4(9)$.

Aristotle. (2011). Excellence quotes. Retrieved from http://www. finestquotes.com/author_quotes-author-Aristotle-page-0.htm

Aviation Glossary. (2010). SOP - standard operating procedures. Retrieved from http://aviationglossary.com/federal-aviationadministration-faadefinition/sop-standard-operating-procedures/

Baker, D. P., \& Krokos, K. J. (2007). Development and validation of aviation causal contributors for error reporting systems (ACCERS). Human Factors: The Journal of the Human Factors and Ergonomics Society, 49(2), 185-199. doi:10.1518/001872007X312432

Bhana, H. (2010). Trust but verify. AeroSafety World, 5(5), 13-1417.

Dismukes, K. (2007). The limits of expertise: Rethinking pilot error and the causes of airline accidents. Aldershot: Ashgate.

Dismukes, R. K., McDonnell, L. K., \& Jobe, K. K. (2000). Facilitating LOFT.debriefings: Instructor techniques and crew participation. International Journal of Aviation Psychology, 10(1), 35-57

Goodman, C. J. (2008). Takeoff and descent of airline employment. Monthly Labor Review, 131(10), 3.

Hale, J. (2011). Helmreich, R. L.A message from the vice president on flight. Unpublished manuscript. (2000). On error management: Lessons from aviation. British Medical Journal, 320 (7237), 781-785.

Helmreich, R. L., Merritt, A. C., \& Wilhelm, J. A. (1999). The evolution of crew resource management training in commercial aviation. International Journal of Aviation Psychology, 9(1), 19-32.

Hughes, D. (1995). FAA may make AQP mandatory. Aviation Week \& Space Technology, 142(3), 27.

Huntzinger, D. (2006). In the PINC. Business \& Commercial Aviation, 98(1), 42. 
Klinect, J. R., Helmreich, R., Murray, P., \& Merritt, A. (2003). Line operations safety audit (LOSA): Definition and operating characteristics. In Proceedings of the 12th International Symposium on Aviation Psychology, 663-668.

National Transportation Safety Board. (2010). Runway side excursion during attempted takeoff in strong and gusty crosswind conditions, continental airlines flight 1404, boeing 737-500, N18611, Denver, CO, December 20, 2008. Aviation Accident Report, AAR-10/04

Pasztor, A. (2009). Safety officials probe American airlines jet's botched landing. Wall Street Journal - Eastern Edition, 254(141), A6.

Quinion, M. (2008). Ologies and isms: A dictionary of word beginnings and endings. Retrieved from http://www.thefreedictionary.com/ quandaries
Rigner, J., \& Dekker, S. (2000). Sharing the burden of flight deck automation training. International Journal of Aviation Psychology, 10(4), 317-326.

Sukenik, N. (1998). Coordination in the cockpit: A game-theory view of standard operation procedures. International Journal of Aviation Psychology, 8(4), 405.

Trumbull, M. (2009). Northwest Airlines flight leads to probe of pilot professionalism. Christian Science Monitor, 90.

Trumbull, M. (2010). Probe blames pilot errors for Colgan Air's Buffalo crash. Christian Science Monitor.

U.S. Navy. (2010). Military requirements for senior and master chief petty officer. Retrieved from http://www.tpub.com/content/advancement/ 14148/css/14148_61.htm 